\title{
Computational Intelligence
}

\author{
Matthew N. O. Sadiku, PhD, P.E. \\ Justin Foreman, PhD \\ Sarhan M. Musa, PhD
}

Prairie View A\&M University/Prairie View, TX, United States of America

Doi: 10.19044/esj.2018.v14n21p56 URL:http://dx.doi.org/10.19044/esj.2018.v14n21p56

\begin{abstract}
Computational intelligence (CI) refers to recreating human-like intelligence in a computing machine. It consists of a set of computing systems with the ability to learn and deal with new situations such that the systems are perceived to have some attributes of intelligence. It is efficient in solving realworld problems which require reasoning and decision-making. It produces more robust, simpler, and tractable solutions than the traditional techniques. This paper provides a brief introduction to computational intelligence.
\end{abstract}

Keywords: Computational, artificial, intelligence, soft computing

\section{Introduction}

Computational intelligence (CI) is the study of the design of intelligent systems. A system is regarded as "intelligent" only if it satisfies learning and decision-making requirements. It is familiar that the best-known manifestation of intelligence is human intelligence. The characteristic of "intelligence" is usually attributed to humans so that $\mathrm{CI}$ is a way of performing like human beings and using human-like reasoning, i.e. it uses inexact and fuzzy knowledge. Thus, the goal of CI is to recreate human-like intelligence in a human-made machine.

The term "computational intelligence" was coined by John McCarthy in 1956. The ongoing worldwide computerization has created new opportunities for researchers. All branches of science and art have become computational: computational biology, computational physics, computational chemistry, computational ecology, computational linguistics, computational electromagnetics, computational finance, computational mechanics, computational social science, computational epistemology, computational intelligence, and so on.

CI uses a combination of five main complementary techniques: (1)fuzzy logic which enables the computer to understand natural language, (2) 
artificial neural networks which permit the system to learn by operating like the biological one, (3) evolutionary computing, which is based on the process of natural selection, (4) learning theory, and (5) probabilistic methods which help in dealing with uncertainties (Wikipedia, 2017). Some of these CI techniques have their origins in biological systems. The taxonomy of computational intelligence techniques is shown in Figure 1 (Abbas, Zhang \& Khan, 2015).

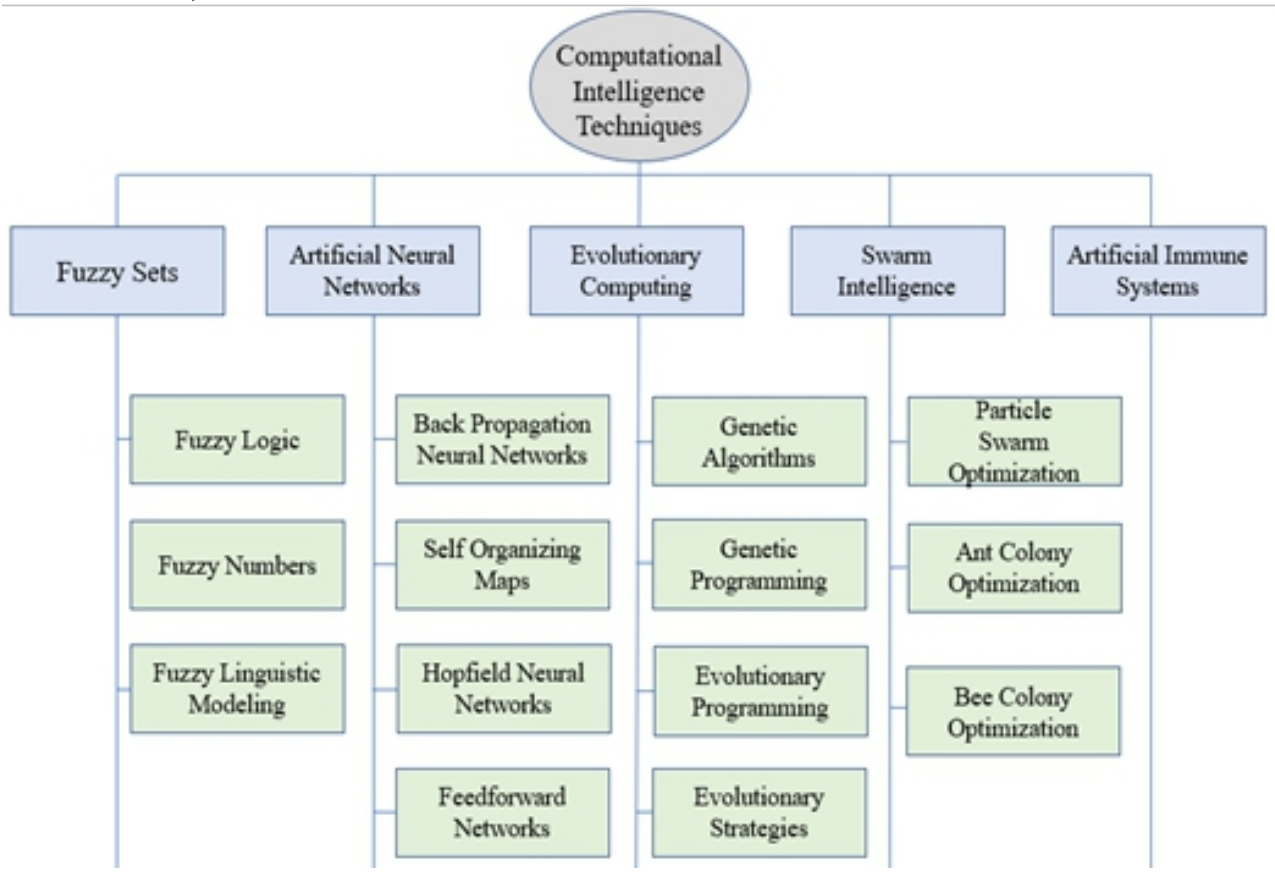

Figure 1 -Taxonomy of computational intelligence techniques (Abbas, 2015)

Although Artificial Intelligence (AI) and Computational Intelligence (CI) seek a similar goal of developing intelligent machines, they are different. $\mathrm{AI}$ is based on hard computing techniques, while $\mathrm{CI}$ is based on soft computing methods. AI operates in a top-down manner, while CI operates in a bottom-up manner. Some regard CI to be a subset or branch of AI.

\section{Applications}

Researchers have used the CI techniques to design intelligent and context-aware algorithms to incorporate the afore-mentioned features. The main applications of $\mathrm{CI}$ include computer science, engineering, business, data analysis, and bio-medicine.

- Business: Computational intelligence techniques have been used to develop business intelligence and solve financial market problems. The techniques have been applied to many areas in business such as marketing, e-commerce, financial management, decision making, 
classification, time series forecasting, and optimization (HernándezJulio et al., 2017) . CI simplifies the problem of predicting future financial markets.

- Music Composition: CI techniques such as neural networks, fuzzy systems, and evolutionary computation provide powerful tools for modeling, learning, and optimization. These techniques have been applied successfully to music composition, which involves musical elements such as timbre, pitch, rhythm, motive, phrase, and chord. The main task of music composition involves deliberating the musical form, creating the melody, and arranging the accompaniment (Liu \&Ting, 2017).

- Social creativity: CI can be used to leverage social creativity. Creative solutions to complex problems faced by organizations come from joint thinking as opposed to traditional views of creativity that focus on the individual as the major contributor of creativity. CI can broaden group participation by linking groups with social networks and can facilitate divergent thinking and inspiration by linking users to huge amounts of online information and social media (Apostolou et al., 2016).

- Medicine: Researchers have used various CI techniques for early diagnosis of heart disease and to improve the heart disease diagnosis accuracy (Jabbar, Deekshatulu \& Chandra, 2015). The application of $\mathrm{CI}$ can be either diagnostic or therapeutic. It can help diagnose diseases and be used in disease prevention (Wiwanitki, 2016). Applying CI techniques in complex medical data can provide better management, faster performance, and higher level of accuracy.

Other areas of applications include games, information systems, biological systems, control systems, power systems (smart grid), economics, oil and gas reservoir, air pollution monitoring, manufacturing systems, corrosion damage assessments, sports, coral reef ecosystems and environmental planning.

\section{Challenges}

There are those who believe that CI is a dead-end, unachievable attempt to recreate intelligence in a computing machine. This belief is based on inconsistent and contradictory goal of CI - while "computational" implies data processing ability, "intelligence" implies the ability to process information (Diamant, 2016).

Each of the various CI techniques has some advantages and suffers from some disadvantages. Therefore, there is no solution methodology that is universally superior. Hybrid methods may generate better results than single methods. 
There have been some issues like security and privacy in data analysis of some sensitive information such as in medical applications. There are also challenges of big data procured from complex healthcare sources (Kalantari et al., 2017).

\section{Conclusion}

Computational intelligence $(\mathrm{CI})$ is a newly evolving research field. It is regarded as a branch of artificial intelligence (AI). It uses various techniques such as artificial neutral networks, evolutionary computation, fuzzy system, support vector machine, deep learning, swarm intelligence, and hybrid methods. As more and more engineering applications use CI techniques, the need to train students in this area increases (Zhang, 2011). The introductory book by Andries Engelbrecht can be helpful (Engelbrecht, 2007).

\section{References:}

1. Computational intelligence, Wikipedia, the free encyclopedia, https://en.wikipedia.org/wiki/Computational_intelligence.

2. Abbas, A., Zhang, L. \& Khan, S.U. (2015). A survey on context-aware recommender systems based on computational intelligence techniques. Computing, 97, 667-690.

3. Apostolou, D. et al. (2016, May). Facilitating creativityin collaborative workwith computational intelligence software. IEEE Computational Intelligence Magazine, 29-40.

4. Diamant, E. (2016). Computational intelligence: are you crazy? Since when has intelligence become computational?Proceedings of IEEE Symposium Series on Computational Intelligence (SSCI). Engelbrecht, A.P. (2007). Computational Intelligence: An Introduction. Chichester, UK: John Wiley \& Sons, $2^{\text {nd }}$ edition.

5. Hernandez-Julio, Y.F., et al. (2017). Framework for the development of business intelligence using computational intelligence and serviceoriented architecture. Proceedings of the 12th Iberian Conference on Information Systems and Technologies (CISTI), 1-7.

6. Jabbar, M.A., Deekshatulu, B.L. \& Chandra, P. (2015). Computational intelligence technique for early diagnosis of heart disease. Proceedings of IEEE International Conference on Engineering and Technology (ICETECH).

7. Kalantari, A., et al. (2017). Computational intelligence approaches for classification of medical data: State-of-the-art, future challenges and research directions. Neurocomputing,1-21.

8. Liu, C.H., \& Ting, C.K. (2017). Computational intelligence in music composition: a survey. IEEE Transactions on Emerging Topics in Computational Intelligence, 1, no. 1, 2017, 2-15. 
9. Wiwanitkit, S., \& Wiwanitkitn,V. (2016). Computational intelligence in tropical medicine. Asian Pacific Journal of Tropical Biomedicine, 6(iv), 350-252.

10. Zhang, M. (2011, August). Experience of teaching computational intelligence in an undergraduate level course. IEEE Computational Intelligence Magazine, 57-59. 